\title{
INFORMATION SECURITY AND MULTISENSOR DATA PROCESSING
}

This issue of Information \& Security is dedicated to one of the most promising areas of contemporary research and development - Multisensor Data Fusion (MSDF). The research community has already adopted common MSDF models and terminology. System developers have reached consensus on main engineering directions, and many commercial off-the shelf data fusion tools are now in use. Why are we focusing our attention on something so closely interrelated with the human cognition practice, and not self-evidently connected with the problems of information security?

As it is well known, the main amount of problems concerning information security arise from the area of signal, data and knowledge processing practice. The emergence of the information society has been inevitably followed by a rapid increase in the implementation of information processing systems. The ascending flood of equipment and sophisticated information systems applications has produced a lack of security for all levels of data processing. The information vulnerability of command and control $\left(C^{2}\right)$ systems became of prime importance. Many system developers started to complain about the insufficient knowledge and expertise. Numerous conferences and symposia on this theme are dedicated to seemingly an endless discussion. But from our point of view, these events are not focused on the exact issue. What is generally neglected is that the essence of all fundamental ideas about information operations and information warfare is the disruption (the protection) of information processing in opposing Command, Control, Communications, Computing and Intelligence $\left(\mathrm{C}^{4} \mathrm{I}\right)$ systems. The core of the information attacks is to produce attrition in the adverse Observe-OrientDecision-Act (OODA) cycle by affecting the performance of $\mathrm{C}^{4} \mathrm{I}$ systems characteristics. The information processing, and more precisely - the multisensor data fusion process, became the "center of gravity" of information operations.

There are two ways to protect our own $\mathrm{C}^{4} \mathrm{I}$ system from adversary invasion. The first one is to shield the information processing hardware and software, giving all responsibility to the "fire-walls". The second is to utilize robust data processing algorithms, providing a new level of sustainability and survivability of the whole system. The first way has been widely discussed in the last decade. Based on this discussion an opinion was formed that the information security will be implemented in ${ }^{4}{ }^{4}$ systems "from outside" - in the form of coding, ciphering or compressing technologies and respective tools. The second approach was left to the $\mathrm{C}^{4} \mathrm{I}$ system designers and engineers. It became their responsibility to assure robustness, sustainability and survivability of the whole information system, in the way they seem appropriate. We do not deny this generic division of the information security problems. We think that now is the right time to include the second approach to $\mathrm{C}^{4} \mathrm{I}$ system protection in the flow of discussions, i.e., to consider information security as development of new sophisticated 
techniques and algorithms for data and information processing.

It is well known that the processing of 'bad' information will contribute to the creation of a bad common picture of situations and threats under consideration. There is no sense to protect poor data, obtained from obsolete sources. Everyone is eager to utilize advanced sensors. They are impressively effective but often vulnerable. The use of multiple sensors, especially those having the capability to measure different physical phenomena (infrared, acoustic, electromagnetic, etc), will improve the robustness of the common picture synthesis and assessment. Thus, the efficient use of multiple sensors can defeat enemy attempts to use jammers, deception or camouflage as components of information operations. Additionally, multiple sensor data fusion provides opportunities to correct errors, produced by each individual source. It is easy to demonstrate that the use of correct models of target behavior and adequate modeling of jamming can significantly improve the robustness of situation assessment processes.

It is clear, that there is no perfect set of techniques and algorithms, which would be optimal under all circumstances. In reality, the mathematical assumptions upon which many of these algorithms are formulated are rarely satisfied. This is one of the main directions of information attacks. They are often successful, because any sophisticated algorithm will produce very poor results, when the input data does not meet the required conditions. So, the effectiveness of the $\mathrm{C}^{4} \mathrm{I}$ systems is sharply dependent on the available a priori knowledge. Unfortunately, the issue of its adequate collection, processing and storing is rarely discussed in the context of information security. We consider this area of contemporary research and development as a promising source for the increase of information security.

Algorithm development for some important applications such as automatic target recognition or identification "friend-foe-neutral" often utilizes specific flow of signals. Based on the specific spectrum patterns, the algorithms are trained to recognize the known targets based on the features extracted from the signals. But today it became obvious, that a thorough discussion on this theme is needed, because for new "stealth" targets there are never enough signals to satisfy the requirement for statistical significance. Besides the numerous methods, which provide the necessary synthetic training data, other techniques must be used to obtain significance for "stealth" pattern recognizers.

Of course, there are many information processing problems related to $\mathrm{C}^{4} \mathrm{I}$ systems resistance to information attacks. Generally speaking, all users seek to improve the security of contemporary $\mathrm{C}^{4} \mathrm{I}$ systems by improving their functional ability to estimate position, velocity, and identity/characteristics of entities. A very promising way to do this is to combine information using multiple target behavior models.

The applications of sensor's data fusion range from situation and threat assessment to smart weapons, automatic target recognition, identification "friend-foe-neutral", and intelligence. Fusion techniques for all these applications are drawn from such advanced disciplines as space-time adaptive signal and image processing, multiple model statistical estimation, neural net pattern recognition, and decisionlevel artificial intelligence processing methods. It is beyond the scope of this issue of I\&S to present all successful multiple sensor data fusion approaches and implementations, related to information security. Our intention is only to illustrate common problems in data fusion and how they are avoided 
or mitigated in particular systems implementation.

We believe this issue will accomplish its mission if two important conclusions about MSDF will appear after acquaintance with the proposed selection of papers. The first one is that in the theoretical and applied considerations of Information Warfare the development of the new sophisticated techniques has to be included. New robust and sustainable algorithms for moving target indication, multiple target tracking, artificial neural network pattern recognition, early warning, genetic algorithms, hybrid intelligent systems for situation and threat assessment, and virtual reality agents and robot control, have to be utilized. The second conclusion is that the knowledge engineering technology becomes of prime importance. It is well known that knowledge is mind's eye in the intelligence, and that the Information Warfare concept is build around the model of the human intuition and decision-making process. No doubt that today this branch of research becomes of a vital importance for the information warfighting concept.

The brief look on proceedings of latest MSDF conferences and workshops, organized by the Information Fusion International Association, shows lists of key research programs for design and development of numerous new technologies and tools. Correct identification of these research areas is crucial for information security in the 21 st century. The basic purpose of this effort is not only "to create explicit, formal catalogs of knowledge that can be used by new data processing systems", but also to create a comprehensive vision for the information security in the 21 st century. It is clear that the developed methods and algorithms are not only creative tools and means, but also parts of the emerging new military warfighting instruments. In this interpretation these methods and algorithms could be considered as smart "weapons", which mission is not only to possess signals, data and knowledge, generating real-time battlefield situation and threat assessments. They are also intended to generate friction in the enemy's $\mathrm{C}^{4} \mathrm{I}$ system, blocking its attempt to do the same. Having this in mind, we believe that the multiple sensor data fusion concept gives general-purpose (i.e. universal) understanding of social security and future warfare. Only the joint interpretation of relations between MSDF and Information Security will form the necessary conceptual frame of reference for understanding the 21 st century environment.

In our attempt to illustrate our vision about information security and multiple sensor data fusion, we compiled this set of articles, which we consider only a representative sample of the huge number of publications. What we are trying to accomplish with this volume is not so much to present particular problem solutions in depth, as to mark the diversity and interdisciplinarity of the research area.

The first article in this edition is devoted to the problem of optimization of the Multi-Source Data Fusion system for Integration on the Canadian Patrol Frigate. Halifax Class Canadian Patrol Frigates and CP-140 (Aurora) fixed wing aircraft are planned to be upgraded within the next decade to be able to deal with far more demanding threat and mission environments of today and the future than when these platforms were designed. All levels of data fusion, resource management and imaging decision support capabilities, and their integration within a generic real-time knowledge base system are considered. The paper describes the efforts towards restructuring and optimizing the proof-of-concept MSDF algorithms to build and demonstrate a real-time prototype which will be ready for integration on the existing platforms and can perform real-time tracking and identification by the end of the year 2000. This paper is an excellent example of successful implementation of MSDF technology for 
overall information security increase in a large complex system.

In the next paper the integration of topographical and topological data in the estimation of the actual traffic situation on airports is studied. The automatic estimation of actual traffic situation on airports has become more and more important with the increase of the security of traffic flow. A method to model and to integrate the airport topography and topology into the traffic situation estimation process is presented in the paper. A filtering algorithm based on the advanced Interacting Multiple Model approach to hybrid systems estimation is proposed. It performs better than the known solutions, and provides an opportunity to rise the public safety in the complex airport area situations. This paper shows how MSDF can improve the security of air traffic control.

The next two papers treat the relation between human decision making and the fusion of information from multiple sources. The authors of the first of the two papers use the concept of integration of information processing functions by humans (instinctual behavior, intuition, motivational and emotional effects, rational decision making) as an useful paradigm to be followed by designers of MSDF architectures and algorithms. The resulting concept of computational intelligence provides for a holistic approach to design and integration of methods and algorithms for information fusion. The authors describe the application of computational intelligence to the fusion of data and information in two studies of early warning. The emphasis is on the power of soft-computing methods in designing early warning architectures pertinent to forecasting events in complex dynamical systems. The parallel with human decision making is found useful in dealing with incomplete and imprecise information on processes on which we have no or limited a priori knowledge.

The second of the two papers threats human intuition from a different perspective. It compares human intuition with computer based decision-making systems. The authors study performance of humans and computer algorithms in the task of classifying airborne targets according to their threat status and the appropriate response from an Integrated Air Defense System. Their results show that partial disclosure of the deterministic algorithm used to classify targets made the classification task even more difficult, contrary to intuition. The inadequacy of intuition is considered as a compelling reason for using specialized methods to design decision support systems.

The last paper in the first group presents an IMMPDA filtering algorithm for radar management and tracking maneuvering targets in the presence of false alarms and Standoff Jammer. The performance of the designed algorithm is evaluated by Monte Carlo simulation. The obtained results demonstrate that the tracking filter satisfies the performance restriction on a maximum allowed track loss posed by the benchmark problem. The reported results provide a glimpse on ongoing studies in this area, as well as on directions of further investigation. When you get acquainted with this paper you will realize how MSDF technology could be successfully utilized in Electronic Warfare.

The second group of papers presents Bulgarian MSDF R\&D program and activities. A brief historical view, chosen strategy and achievements in the field of MSDF, and current joint R\&D projects are presented.

The first paper of authors form the Central Laboratory for Parallel Processing at the Bulgarian Academy of Sciences presents an effective Doppler-filtering algorithm using higher-order statistics. 
An algorithm for moving target selection in the presence of clutter and wideband jammer is presented. The algorithm performance is investigated by the means of Monte Carlo simulation analysis. The obtained result shows how the information included in the reflected signal could be effectively exploited for development of robust algorithms.

Tracking filters for radar systems with correlated measurement noise is the subject of consideration in the next article. Tracking filter for systems with colored measurement noise is developed. A new technique for adaptive evaluation of the algorithm parameters is proposed. The realized algorithm is incorporated into Interacting Multiple Model schemes for tracking maneuvering objects. A substantial improvement in velocity and acceleration estimation is achieved. Obviously, the robustness and security of such algorithms will be higher because of their capability to work in uncertain environment.

An Interacting Multiple Model algorithm for stochastic systems control is presented in the next paper. The overall system control is synthesized as a probabilistically weighted sum of the control processes from separate regulators working in parallel. These regulators are synthesized for each model from the uncertainty domain. The simulation results demonstrate that the IMM control algorithm provides better results in the presence of abrupt changes in the parameters than the already utilized similar algorithms. Because of the sustainability and survivability of data processing in conditions of random perturbations, this kind of algorithms is very promising for an increase in information processing security.

Multiple hypothesis tracking using Hough transform track detector is proposed and evaluated in the next paper. Uncertainty in the measurements is managed by the usage of asynchronous multiple hypothesis algorithms. At the cost of delayed track detection, this algorithm shows remarkable good performance and noise resistance. The inclusion of this paper in the issue has been motivated by the successful implementation of the multiple hypothesis approach. This approach is one of the most important instruments for fighting uncertainty in hostile environment (jammers, false alarms, closely spaced targets, crossing trajectories, etc.). By using the a priori information about the situations and threats that will appear, multiple hypothesis approach gives an opportunity to raise the reliability, and thus the security, of data processing. An interesting feature of the study is the combined use of multiple hypothesis tracking with Hough transform track initiator.

Maneuvering ship tracking, especially ship collision avoidance, is a problem of a great practical and theoretical interest. Real-world tracking applications meet a number of difficulties caused by the presence of different kinds of uncertainty due to the unknown or not precisely known system model and random processes' statistics or because of their abrupt changes. These problems are especially complicated in the marine navigation practice, which needs a high level of security of the vessels traffic control. A solution of these problems is presented in the paper. A new ship model is derived after an analysis of basic hydrodynamic models. This model is implemented in a new version of the Interacting Multiple Model tracking algorithm - the most cost-effective multiple model algorithm for hybrid estimation. The performed Monte Carlo simulation shows that the model fits available data excellently. The obtained good estimation performance demonstrates that this algorithm could be successfully implemented in collision avoidance system for reliable real-time data processing. 
For readers, interested to learn more about MSDF, four fundamental books are presented in this volume: Multisensor Data Fusion by Edward Waltz and James Llinas, Information Warfare Principles and Operations by Edward Waltz, Bayesian Multiple Target Tracking by Lawrence Stone, Carl Barlow, Thomas Corwin, and Multitarget/Multisensor Tracking: Applications and Advances (Volume III) by Yaakov Bar-Shalom and William Blair. The fifth book - Sensors for Peace: Applications, Systems and Legal Requirements for Monitoring in Peace Operations edited by Jurgen Altmann, Horst Fisher and Henny van der Graaf - provides a multidimensional study of the connection between sensor system technologies and important international security issues such as the efficiency of peacekeeping operations. We find these books very useful not only for students and Ph.D. applicants, but also for specialists who are not familiar with the foundations of MSDF, but are interested in further applications and have a good mathematics background. For the beginners, a small set of information fusion terminology is given. Additionally, a short list of introductory publications is proposed. For more information and references a sample of Internet links is provided, as well as a schedule of events of the International Society of Information Fusion.

We hope this issue will help to develop new interrelations within different areas of science community. The common interest in solving Information security problem using MSDF technologies could provide new opportunities for fruitful cooperation and consideration of future joint R\&D projects.

\section{$\underline{\text { BACK TO TOP }}$}

(C) 1999, ProCon Ltd, Sofia

Information \& Security. An International Journal

e-mail: infosec@mbox.digsys.bg 